Rechtsmedizin 2010 • 20:85-90 DOI 10.1007/s00194-010-0659-3

Online publiziert: 4. März 2010

๑) Springer-Verlag 2010
M.A. Rothschild ${ }^{1}$ - B.P. Kneubuehl ${ }^{2}$

${ }^{1}$ Institut für Rechtsmedizin, Universitätsklinikum Köln (AöR), Köln

${ }^{2}$ Institut für Rechtsmedizin, Universität Bern, Bern
Wie alle naturwissenschaftlichen Vorgänge lassen sich auch ballistische Gegebenheiten und wundballistische Prozesse mithilfe physikalischer Gesetze erklären und mit der Mathematik als Sprache beschreiben. Auch wenn nach Patscheider (mündl. Mitteilung) - bezogen auf Schussbefunde in rechtsmedizinischen Prosekturen - scheinbar jeder Schuss anders ist, sind die Phänomene mit den Gesetzen der Naturwissenschaft erklärbar. Umso mehr erstaunt, dass gerade in der Wund-/Ballistik eine derart große Zahl an Irrtümern und Fehlinterpretationen vorherrscht. Nachfolgend wird eine Auswahl besonders häufig anzutreffender Fehldeutungen vorgestellt und korrigiert.

\section{Simulanzien für \\ Schussexperimente}

Es ist ein weit verbreiteter Irrtum, dass zur wissenschaftlichen Beurteilung von Schusswirkungen auf lebende oder tote Tiere oder sogar menschliche Leichen geschossen würde. Aus ethischen und wissenschaftlichen Gründen werden in der wundballistischen Forschung standardisierte Simulanzien verwendet, die sich beim Beschuss wie menschliches Körpergewebe mit einer Temperatur von $37^{\circ} \mathrm{C}$ verhalten. Seit vielen Jahren haben sich hierzu Glyzerinseife und Gelatine bewährt, die bei bestimmten Beschusstemperaturen dem Geschoss gegenüber ein sehr ähnliches Verhalten aufweisen wie weiche Gewebe (ausgenommen die Lunge).

\title{
Irrtümer in der Wundballistik
}

\section{Aus ethischen und wissenschaftlichen Gründen werden standardisierte Simulanzien verwendet}

Aufgrund der Homogenität dieser Simulanzien lassen sich Schüsse reproduzieren, Gesetzmäßigkeiten durch gezielte Variation von Parametern erkennen und durch Vergleich von realen Verletzungsfällen mit Beschädigungen am Simulans die Resultate von Schussversuchen auf den Menschen übertragen. Auch wenn sich das Simulationsmaterial im Hinblick auf Elastizität, Plastizität, Viskosität, Energieübernahmefähigkeit, Widerstand etc. auf ganzer Länge des Schusskanals konstant verhält, wohingegen im menschlichen Körper Gewebe mit sehr unterschiedlichen Eigenschaften (z. B. Muskulatur, Lunge) aufeinanderfolgen, hat dies auf das ballistische Verhalten des Geschosses keinen wesentlichen Einfluss. Der Hauptgrund hierfür liegt in den hohen Geschwindigkeiten, mit denen sich physikalisch-ballistische Prozesse abspielen. Aus diesem Grund verhalten sich auch an sich so unterschiedliche Materialien wie Gelatine und Seife beim Beschuss sowohl im Hinblick auf die Energie, die das Geschoss auf das Simulans überträgt, als auch im Hinblick auf das Deformationsverhalten des Geschosses gleichartig.

Die Wahl des zu verwendenden Simulans wird durch den Versuchszweck bestimmt: Die elastische Gelatine eignet sich wegen ihrer Transparenz v. a. für das Erfassen von Bewegungsabläufen, z. B. mit Hochgeschwindigkeitskameras. Zudem ist Gelatine vergleichsweise leicht selbst herstellbar und damit preisgünstig; die Auto- ren haben gute Erfahrungen mit Gelatine „Type Ballistic 3 Photographic Grade“ (Gelita AG; Gelstärke 245-275 Bloom, Viskosität 3,4-4,6 mPa•s, pH 4,7-5,7). Bezüglich des Mischungsverhältnisses von Wasser zu Gelatine und der Temperatur beim Beschuss existieren international verschiedene Normierungen; hier bestehen gute Erfahrungen mit dem Standard von Fackler u. Malinowski [2]: 10\%ige Gelatine bei $4^{\circ} \mathrm{C}$. Zur Simulation des Knochenschusses können synthetische Knochen (hergestellt aus Polyurethanschichten) in natürlichen oder geometrischen Formen mit in die Gelatine eingegossen werden (Synbone AG, Malans, Schweiz, http:// www.synbone.ch). Periost und Knochenmark werden dabei ebenfalls nachgebildet [9]. Entsprechend gibt eine mit Gelatine gefüllte Polyurethankugel ein ausgezeichnetes Kopfmodell [25] für Beschussversuche ab.

Glyzerinseife (z. B. Permatin AG, Stein am Rhein, Schweiz, http://www.permatin.ch) lässt sich demgegenüber leichter handhaben, erlaubt wegen ihrer Plastizität Volumenmessungen des Schusskanals, wodurch sich (wegen des linearen Zusammenhangs zwischen Energieabgabe und gebildetem Volumen) Energieverläufe leicht bestimmen lassen. Zudem lässt sich Seife länger gebrauchsfähig lagern.

\section{Einschussmorphologie: Entsteht der "Schürfsaum" durch Schürfung?}

Einschussverletzungen durch senkrecht auftreffende Vollmantelmunition weisen in der Regel eine Reihe typischer Befunde auf: Im Zentrum liegt der zentrale 
Substanzdefekt, daran anschließend findet sich ein zirkulärer Abstreifring, dann um diesen herum ein "Schürfsaum“ und schließlich außen der Dehnungssaum.

Tatsächlich sieht der „Schürfsaum“ so aus, als sei er durch das Einstülpen der Haut durch das eindringende Projektil und anschließende Abschürfen der Geschossoberfläche auf der Haut am Einschuss entstanden. Aber schon Sellier [22, 23, 24] mit seinen Hochgeschwindigkeitsuntersuchungen sowie Pollak [17], Pollak u. Ropohl [18] und Thali et al. [25] wiesen die tatsächliche Verursachung dieser ringförmigen Oberhautverletzungen nach: Beim schlagartigen Auftreffen des Projektils werden innerhalb von Mikrosekunden Oberhautpartikel und Anteile des Coriums unter und neben dem Geschosskopf mit hoher Geschwindigkeit radial weggeschleudert.

\section{(7) Der ringförmige Gewebeverlust entsteht durch energiereiche Kontusion}

Durch diese energiereiche Kontusion entsteht ein ringförmiger Gewebeverlust. Zusätzlich wird durch die radiale Beschleunigung des Gewebes beim Eindringen des Projektils die Haut im Einschussbereich vergleichbar der temporären Höhle kurzfristig überdehnt. Die Folge sind kleinste, radial angeordnete Überdehnungsrisse der Oberhaut, teilweise flankiert von mit freiem Auge sichtbaren Einreißungen. Das Resultat ist eine durch Überdehnungseffekte entstandene, 2-3 mm breite Hautveränderung, die bei Verstorbenen leicht an der braunroten Vertrocknung (daher auch synonym: Vertrocknungssaum) erkennbar ist. Da der Effekt im Wesentlichen kontusionsbedingt ist, muss der „Schürfsaum“ korrekterweise als Kontusionsring bezeichnet werden.

\section{Unterschied zwischen Querschläger und Abpraller}

Die beiden Begriffe Querschläger und Abpraller werden häufig fälschlich synonym verwendet, beschreiben aber zwei unterschiedliche Vorgänge: Beim Querschläger handelt es sich um ein Projektil, das mit seiner Längsachse quer zur Bewegungsrichtung ,aufschlägt“. Die Grün- de hierfür können z. B. in einem systembedingten instabilen Geschossflug, dem Durchdringen eines Zwischenmediums mit Verlust der Stabilität oder aber auch in einem Abprallvorgang liegen. Ein Abpraller (Ricochet) bezeichnet ein Projektil, das auf seiner Flugbahn ein festes oder flüssiges Medium berührt und daduch eine Änderung in seiner Flugbahn und Fluglage erfährt. Durch diesen Kontakt kann sich sein Anstellwinkel (Winkel zwischen Projektilachse und Flugrichtung) so vergrößern, dass es beim Auftreffen auf ein Objekt als Querschläger erscheint. Abgeprallte Geschosse können auf ihrer weiteren Flugbahn durchaus wieder in eine normale Fluglage (Geschossachse in Flugrichtung) zurückfinden, wobei allerdings sowohl die Spitze als auch das Heck vorn möglich sind. Ein Abpraller kann somit zu einem Querschläger werden, muss es aber nicht.

Im Englischen spricht man bei einem Querschläger von einem „keyhole“ bzw. „keyholing“. Der Abpraller ist ein „ricochet“. Im Französischen gibt es diese ballistisch sinnvolle Unterscheidung allerdings nicht; hier werden beide Begriffe jeweils synonym als „ricochet“ verwendet.

\section{Waffen- und Munitionsbezeichnungen}

Nach dem Waffengesetz werden bei Schusswaffen Kurz- und Langwaffen voneinander unterschieden. Langwaffen sind solche, bei denen Lauf und Verschluss in geschlossener Stellung insgesamt länger als $30 \mathrm{~cm}$ sind und deren kürzeste bestimmungsgemäß verwendbare Gesamtlänge $60 \mathrm{~cm}$ überschreitet; Kurzwaffen sind alle anderen Schusswaffen [Anlage 1 Nr. 2.5 zu $\$ 1$ Abs. 4 Waffengesetz (WaffG)]. Bei den Kurzwaffen handelt es sich überwiegend um Pistolen und Revolver. Der Revolver unterscheidet sich von den Pistolen und Gewehren dadurch, dass bei ihm das Patronenlager vom Lauf getrennt ist und bei den anderen mit dem Lauf eine Einheit bildet. Bei den Langwaffen werden im zivilen Bereich hauptsächlich Büchsen und Flinten unterschieden, Erstere mit einem gezogenen Lauf (mit Zügen und Feldern), Letztere mit einem glatten Lauf. Nach einer Handhabungsdefinition kann man Faust- und Handfeuerwaffen voneinan- der unterscheiden: Faustfeuerwaffen lassen sich mit einer Hand bedienen, während man zum Schießen mit Handfeuerwaffen beide Hände benötigt (zumeist Langwaffen).

Die Bezeichnung von Patronenmunition ist häufig eine Mischung aus Kaliberangabe, Hülsenlänge und/oder Zusatzbezeichnung. Für die Bezeichnungen existieren zwei internationale Standards nach der Commission Internationale Permanente pour l'Épreuve des Armes à Feu Portatives (C.I.P) und des Sporting Arms and Ammunition Institute (SAAMI). Hiernach wird das Kaliber in Millimetern oder „inch“ angegeben. Bei den Angaben in Inch wird auf das „o,“ verzichtet (z. B. 380 anstatt o,380). Auch die Zusatzbezeichungen folgen diesem Standard (z. B. Spl., Mag. etc.). Die Kaliberbezeichnung deckt sich nicht immer mit dem effektiven Laufkaliber der Waffe. So haben Waffen des angloamerikanischen Kalibers 38 (380, 38 S\&W, 38 Spl.) ein Laufkaliber von $9 \mathrm{~mm}$ (und nicht 0,38 inches, was 9,65 mm entspricht).

Bei Schrotmunition bezeichnet das Kaliber den Durchmesser des Laufs, nicht des einzelnen Schrots [7]. Grundlage für die Kaliberbezeichnung ist 1 englisches Pfund $(453,6$ g) Weichblei: Kaliber 12 bedeutet der Durchmesser, den man je Kugel erhält, wenn man aus 1 Pfund Weichblei 12 gleich große Kugeln gießt; Kaliber 16 bedeutet der Durchmesser, den jede von 16 gleich großen Kugeln hat, die man aus 1 Pfund Weichblei gießt. Die Besonderheit liegt darin, dass die kleinere Kaliberzahl den größeren Patronen- und damit Laufdurchmesser bezeichnet (Kaliber 12 ist größer als Kaliber 16). Die zweite Zahl in der Kaliberbezeichnung gibt die Länge der entfalteten (abgeschossenen) Hülse an (z. B. Kaliber 12/70=70-mm-Hülsenlänge, $12 / 76=76-\mathrm{mm}$-Hülsenlänge).

\section{Gefährlichkeit des Schusses steil in die Luft}

Zur Beurteilung der Frage, ob ein steil in die Luft geschossenes Projektil in der Lage ist, beim Zurückfallen in einen Körper einzudringen, muss seine so genannte Fallschirmgeschwindigkeit $\mathrm{v}$ berechnet werden [10], bei der sich die Erdbeschleunigung und die Verzögerung durch 
den Luftwiderstand die Waage halten. Diese Geschwindigkeit hängt neben der Erdbeschleunigung g vom Luftwiderstandsbeiwert $\mathrm{c}_{\mathrm{w}}$, von der Querschnittsbelastung $q$ und der Luftdichte $\rho$ ab.

$$
v=\sqrt{\frac{2 \cdot q \cdot g}{c_{w} \cdot \rho}}
$$

In den meisten Fällen wird diese Geschwindigkeit allerdings erst kurz vor Erreichen der Mündungsebene oder gar erst danach erreicht. Sie dient jedoch als Schätzwert für die Geschwindigkeit zurückfallender Geschosse.

Die ballistischen Daten verschiedener fallender Geschosse sind in $\bullet$ Tab. 1 zusammengefasst. Die Energiedichte beim Auftreffen (ED) von aus Luftdruckwaffen verschossenen Diabologeschossen oder aus Flinten verfeuerter Schrotmunition (Schrot $\leq 5 \mathrm{~mm}$ ) liegt deutlich unter der Grenzenergiedichte zum Durchschlagen von Haut $\left(0,1 \mathrm{~J} / \mathrm{mm}^{2}\right)$ oder Knochen $\left(0,2 \mathrm{~J} / \mathrm{mm}^{2}\right)$; dies lässt derartige Schüsse in die Luft als nichtlebensbedrohlich einstufen. Anders sieht es aber mit allen anderen durch Faust- oder Handfeuerwaffen steil in die Luft verschossenen Projektilen aus. Hier reicht die Energie in jedem Fall aus, um z. B. bei Kopftreffern nicht nur die Haut, sondern auch den Schädel zu durchschlagen.

\section{Wirken Knochensplitter als Sekundärgeschosse?}

Wird ein platter Knochen nahezu senkrecht von einem Geschoss getroffen, ist der Energieverlust des Geschosses mit 60-70 J relativ gering. Diese abgegebene Energie wird primär für die Zerstörungsarbeit beim Knochenbruch aufgewendet und erst in zweiter Linie zum Mitreißen der Knochensplitter. Unter der Annahme, dass die Hälfte der für den Durchschuss aufgewendeten Energie für den Knochenbruch, die andere Hälfte für die Beschleunigung der Splitter verwendet wird und etwa 10 Splitter entstehen, kommt im Durchschnitt jedem Splitter eine Energie von ca. 3,5 J zu. Zudem haben die schussbedingt entstehenden Knochensplitter eine zumeist derart geringe Masse, dass sie trotz der ihnen übertragenen Energie kein

Rechtsmedizin 2010 - 20:85-90 DOI 10.1007/s00194-010-0659-3

C Springer-Verlag 2010

\section{M.A. Rothschild · B.P. Kneubuehl Irrtümer in der Wundballistik}

\section{Zusammenfassung}

In der Wundballistik gibt es eine Vielzahl von tradierten Irrtümern, die jedoch bei objektiver naturwissenschaftlicher Betrachtung rasch ausgeräumt werden können. Als Gewebesimulanzien für wundballistische Experimente sind aus Gründen der Vergleichbarkeit und Nachvollziehbarkeit nur Glyzerinseifen und Gelatine zu verwenden. Bei Einschussverletzungen sollte man den „Schürfsaum" nur noch Kontusionsring nennen, da er nicht durch Schürfung bedingt ist. Abpraller und Querschläger sind nicht dasselbe. Schüsse steil in die Luft sind gefährlich, da die herabfallenden Projektile genügend Energie haben, um in den Kopf einzudringen (Ausnahme Luftdruckwaffen- und Schrotschüsse bis 5-mm-Durchmesser). Knochensplitter können aufgrund der an sie übertragenen relativ geringen Energie nicht als, Sekundärgeschosse" wirken. Mit Schüssen aus Faustoder Handfeuerwaffen kann man keine

\section{Misconceptions in wound ballistics}

\section{Abstract}

In ballistics, especially wound ballistics, a large number of misconceptions and false conclusions concerning findings and their causes exist. On the other hand these misconceptions can be easily corrected by using objective scientific methods and tools. For performing experiments in wound ballistics glycerine soap and gelatine should be used when human soft tissue has to be simulated. Gunshots into the air can be dangerous to life as the falling bullets have enough energy to penetrate skin and bones; exceptions are air gun and shotgun pellets which do not have enough energy to penetrate skin when descending. When a bullet hits bone the bone fragments will not receive enough energy to act as secondary projectiles themselves. Shots from handguns are not able to produce enough power to stop a person or
„Mann-Stopp-Wirkung" erreichen. Schreckschusswaffen sind bei absoluten Nahschüssen gefährlich, weil der Gasstrahl aufgrund seiner hohen Geschwindigkeit eine Energiestromdichte erreicht, die ihn, physikalisch betrachtet, zum Massenstrom und somit zum quasi-soliden Geschoss werden lässt. Die Wirkung eines Schusses ist ein Einzelereignis, das von einer Vielzahl individueller Faktoren abhängt; die Wirksamkeit hingegen beschreibt das grundsätzliche Verletzungspotenzial, das einem Geschoss bzw. System innewohnt. Die Wirksamkeit ist in erster Linie von der Auftreffenergie des Geschosses und der Fähigkeit, diese Energie entlang des Schusskanals abzugeben, abhängig.

Schlüsselwörter

Wundballistik · Schuss · Wirksamkeit · Irrtum . Fehlinterpretation throw a person backwards. Blank shots from alarm pistols are dangerous to life when fired at point blank range: the gas jet exiting the muzzle has such a high velocity that a high energy density will result which gives the jet the character of a solid projectile. The effect of a gunshot is an individual event which depends on a number of factors (e.g. localization of the entrance wound and wound canal, psychological state, intoxication and pathological state of organs). Effectiveness means the wounding potential and is dependent on the energy of the bullet and its ability to transfer the energy along the wound canal.

\section{Keywords}

Wound ballistics - Gunshot - Effectiveness . Misconception · False conclusion 
Tab. 1 Ballistische Daten fallender Geschosse [10]

\begin{tabular}{|c|c|c|c|c|c|c|c|}
\hline Kaliber & Geschoss & $\begin{array}{l}\text { d } \\
(\mathrm{mm})\end{array}$ & $\begin{array}{l}h_{\max } \\
(\mathrm{m})\end{array}$ & $\begin{array}{l}v_{f} \\
(m / s)\end{array}$ & $\begin{array}{l}v_{e} \\
(m / s)\end{array}$ & $\begin{array}{l}E_{e} \\
(J)\end{array}$ & $\begin{array}{l}\mathrm{ED}_{\mathrm{e}} \\
\left(\mathrm{J} / \mathrm{mm}^{2}\right)\end{array}$ \\
\hline $4,5 \mathrm{~mm}$ & Diabolo & 4,5 & 224 & 39,4 & 37,5 & 0,37 & 0,023 \\
\hline Schrot (Pb) & Kugel & 4,0 & 218 & 32,0 & 29,8 & 0,16 & 0,013 \\
\hline Schrot (Pb) & Kugel & 5,0 & 262 & 36,0 & 33,6 & 0,41 & 0,021 \\
\hline Schrot (Pb) & Kugel & 6,0 & 302 & 39,4 & 36,8 & 0,85 & 0,030 \\
\hline 22 L.R. & $\mathrm{PbR}$ & 5,6 & 858 & 81,5 & 76,4 & 7,44 & 0,30 \\
\hline 9 mm Luger & VMR & 9,0 & 1101 & 94,5 & 89,6 & 32,1 & 0,51 \\
\hline 38 Spl. & SWC & 9,0 & 889 & 92,1 & 85,1 & 37,1 & 0,58 \\
\hline 357 Mag. & SWC & 9,0 & 1030 & 92,1 & 86,7 & 38,5 & 0,61 \\
\hline 44 Rem. Mag. & TMF & 11,2 & 1300 & 103,7 & 97,7 & 74,2 & 0,75 \\
\hline $5.56 \mathrm{~mm}$ NATO & VMS & 5,56 & 2300 & 125,9 & 123,7 & 30,6 & 1,26 \\
\hline $7,62 \times 39$ & VMS & 7,62 & 1799 & 119,7 & 110,4 & 48,8 & 1,07 \\
\hline $7,62 \times 51$ & VMS & 7,62 & 2556 & 139,5 & 137,6 & 89,9 & 1,97 \\
\hline $8 \times 68$ & VMR & 8,0 & 3059 & 167,1 & 161,0 & 184,7 & 3,67 \\
\hline \multicolumn{8}{|c|}{$\begin{array}{l}\mathrm{d} \text { Geschossdurchmesser, } \mathrm{E}_{\mathrm{e}} \text { Auftreffenergie, ED Energiedichte beim Auftreffen, } \mathrm{h}_{\max } \text { maximale Steighöhe, } \mathrm{v}_{\mathrm{e}} \\
\text { effektive Auftreffgeschwindigkeit, } \mathrm{v}_{\mathrm{f}} \text { Fallschirmgeschwindigkeit.SWC, semi-wadcutter", TMF Teilmantelflach- } \\
\text { kopf, VMS Vollmantelspitzkopf, VMR Vollmantelrundkopf, } \mathrm{Pb} \text { Blei, PbR Bleirundkopf. }\end{array}$} \\
\hline
\end{tabular}

\section{Tab. 2 Ballistische Daten zu Rückwurf- und Drehgeschwindigkeiten [11]}

\begin{tabular}{lllllll} 
Kaliber & $\begin{array}{l}\mathbf{m}_{\mathbf{G}} \\
(\mathbf{g})\end{array}$ & $\begin{array}{l}\mathbf{v}_{\mathbf{G}} \\
(\mathbf{m} / \mathbf{s})\end{array}$ & $\begin{array}{l}\mathbf{E} \\
(\mathbf{J})\end{array}$ & $\begin{array}{l}\mathbf{p}_{\mathbf{G}} \\
(\mathbf{N} \cdot \mathbf{s})\end{array}$ & $\begin{array}{l}\mathbf{v}_{\mathbf{K}} \\
(\mathbf{c m} / \mathbf{s})\end{array}$ & $\begin{array}{l}\mathbf{v} \\
\left(\mathbf{s}^{-1}\right)\end{array}$ \\
\hline 9 mm Luger & 8,0 & 350 & 490 & 2,8 & 3,5 & 0,009 \\
\hline 45 Auto & 14,9 & 260 & 505 & 3,9 & 4,9 & 0,012 \\
44 Rem. Mag. & 15,6 & 440 & 1510 & 6,9 & 8,6 & 0,022 \\
308 Winchester & 9,5 & 830 & 3270 & 7,9 & 9,9 & 0,025 \\
12/70 Flintenlaufgeschoss & 31,5 & 400 & 2400 & 12,6 & 15,8 & 0,038 \\
$\begin{array}{l}\text { E Geschossenergie, } \mathrm{m}_{\mathrm{G}} \text { Geschossmasse, } \\
\text { keit, } \mathbf{v}_{\mathrm{K}} \text { Rückwurfgeschwindigkeit. }\end{array}$ & $\mathbf{p}_{\mathrm{G}}$ Geschossimpuls, v Drehgeschwindigkeit, $\mathbf{v}_{\mathrm{G}}$ Geschossgeschwindig- \\
\hline
\end{tabular}

wesentliches Zerstörungspotenzial haben dürften.

Beim Röhrenknochen ist die übertragene Energie deutlich größer (bis gegen 300 J bei einem Femur, durchschossen mit einem Gewehrgeschoss). Wird ein solcher Röhrenknochen von einem Geschoss, dessen Kaliber kleiner als der Durchmesser des Knochens ist, relativ mittig getroffen, erfolgt der schussbedingte Knochenbruch in erster Linie durch den sich im Knochenmark aufbauenden hydraulischen Druck. Hierbei werden die Knochensplitter sowohl in Schussrichtung als auch in die entgegengesetzte Richtung weggeschleudert. Aber auch hier reicht die an die Knochensplitter übertragene Energie nicht aus, diese so zu beschleunigen, dass hierdurch eigene Schusskanäle resultieren (Überlegung analog oben).

- Knochensplitter fliegen hinter dem Geschoss im Vakuum der sich bildenden temporären Höhle.
Dennoch scheinen häufig Knochensplitter innerhalb der Weichteile seitlich vom Schusskanal zu „stecken“. Durch die schussbedingte Knochenverletzung werden Knochensplitter abgesprengt, die dem Geschoss in die temporäre Höhle nachfolgen, in der sie dann - oft recht weit vom Knochen entfernt - zu liegen kommen. Kollabiert die temporäre Wundhöhle anschließend wieder, werden die Knochensplitter in die sich bildenden Wundtaschen eingeschlossen und scheinen danach fernab des eigentlichen (bleibenden) Schusskanals am Ende eigener „Schusskanäle“ zu liegen. Diese Überlegungen werden durch Hochgeschwindigkeitsaufzeichnungen bestätigt, die zeigen, dass die Knochensplitter nach dem Durchschuss dem Geschoss folgen und nicht voraneilen.

Lediglich bei größeren Knochenfragmenten und bei niedrigen Geschwindigkeiten kann ein etwas anderer Mechanismus vorliegen. Hier kann es durch Kontakt mit dem Geschoss zu einem schar- nierartigen Wegklappen des Knochenstücks kommen, das u. U. sogar in das unmittelbar benachbarte Weichteilgewebe hineingedrückt werden könnte. Die Bezeichnung Sekundär-"Geschoss" erscheint aber auch hier nicht angebracht.

\section{Mann-Stopp-Wirkung}

Mit gewisser Hartnäckigkeit wird immer wieder vorgetragen, dass mit energiereichen Schüssen aus Faust- oder Handfeuerwaffen eine erwachsene Person umgestoßen bzw. zurückgeschleudert werden kann („stopping power“ oder „Aufhaltekraft"). Unterstützt wird diese irrige Annahme durch zahlreiche Darstellungen in Filmen, bei denen Getroffene zurückgeschleudert und dabei noch durch Türen und sogar Wände gestoßen werden. Schon mit einfachen physikalischen Berechnungen kann man aufzeigen, dass es bei derartigen Waffensystemen eine solche Mann-Stopp-Wirkung nicht geben kann [5]. Mithilfe des Impulserhaltungssatzes

$$
\mathrm{m}_{1} \bullet \mathrm{v}_{1}=\mathrm{m}_{2} \bullet \mathrm{v}_{2}
$$

wird schnell verständlich, dass beispielsweise ein $8 \mathrm{~g}$ schweren Geschoss $\left(\mathrm{m}_{1}\right)$ selbst bei zweifacher Schallgeschwindigkeit $\left(\mathrm{v}_{1}\right)$ und unter der Annahme eines Steckschusses und somit vollständiger Energieübertragung (vollständig inelastischer Stoß) einen $80 \mathrm{~kg}$ schweren Menschen $\left(\mathrm{m}_{2}\right)$ aufgrund der 10.000-mal geringeren Masse kaum wesentlich zurückschleudern $\left(\mathrm{v}_{2}\right)$ können wird. In • Tab. 2 sind die ballistischen Daten, einschließlich der Rückwurf- und Drehgeschwindigkeiten, für verschiedene Kaliber aufgeführt. Selbst bei einem Treffer mit einem Flintenlaufgeschoss wird man bei einem (stehenden) Beschossenen aufgrund des Impulses kaum mehr als einen Ausfallschritt nach hinten erwarten dürfen.

Berücksichtigt man zudem, dass hier nicht nur das dritte Newton-Axiom (actio gleich reactio) für frei im Raum befindliche Körper zu beachten ist, sondern der Getroffene zum Zeitpunkt des Schusserhalts steht bzw. auf den Schützen zuläuft, wird klar, dass man eine Mann-StoppWirkung allein aufgrund des Impulses durch das Projektil selbst bei einem Steckschuss kaum erwarten können wird. 


\section{Sofortige absolute Handlungsunfähigkeit}

Eine besondere Herausforderung stellen für die Polizei Situationen dar, in denen eine unmittelbare Bedrohungslage durch eine Person gegen eine oder mehrere andere Personen vorliegt. In der konkreten Situation muss der Polizist einerseits das Leben der bedrohten Person schützen, andererseits versuchen, hierfür das schonendste, am wenigsten progressive Mittel einzusetzen. Die Entwicklung des „taser“ stellt in jüngster Zeit in diesem Zusammenhang für bestimmte Einsatzsituationen eine interessante Alternative dar $[1,12]$.

Der Grad der Entscheidung und die Wahl des Einsatzmittels werden aber in extremen Situationen immer kleiner werden müssen. Bei einer klassischen Geiselnehmersituation mit unmittelbarer vitaler Bedrohung einer Geisel, etwa durch eine gegen den Kopf der Geisel gerichteten Schusswaffe, muss der polizeiliche Präzisionsschütze über die Wirkung seines Schusseinsatzes absolut sicher sein. Wenn es keine andere Möglichkeit der Konfliktlösung mehr gibt und das Leben der Geisel unmittelbar bedroht ist, muss er sicher sein, dass sein Schuss den Geiselnehmer derart beeinträchtigen wird, dass dieser nicht einmal mehr den Abzug seiner Waffe betätigen kann.

Eine solche sofortige absolute Handlungsunfähigkeit ist nur zu erwarten, wenn das Zentralnervensystem des Getroffenen ausgeschaltet wird. Dies ist über eine Schusseinwirkung nur zu erreichen, wenn wesentliche zentrale Anteile des Gehirns bzw. des oberen Halsmarks verletzt werden. $\mathrm{Zu}$ diesen Strukturen gehören Stammganglien, Di- und Mesencephalon, Cerebellum, Hirnstamm, Pons und Medulla oblongata $[4,6,21]$. Daraus folgt, dass eine sichere sofortige, absolute Handlungsunfähigkeit nur mit der Beibringung unmittelbar tödlicher Verletzungen zu erreichen ist. Zwar kann auch ein Schuss gegen übrige Körperregionen zu einem sofortigen Kollaps der getroffenen Person führen, ohne sie zu töten, z. B. psychogen aufgrund einer Treffererwartung. Allerdings ist der Eintritt dieser Wirkung unsicher, nichtvorhersehbar und von vielen Faktoren abhängig. Und es gibt in der Fachliteratur zahlreiche Beispiele dafür,

Tab. 3 Energiedichten von Schreckschusswaffen in Abhängigkeit von Laufattrappenlänge und Mündungsabstand. (Mod. nach [11])

\begin{tabular}{|c|c|c|c|c|c|}
\hline \multirow[t]{2}{*}{ Kaliber } & \multirow[t]{2}{*}{ Waffentyp } & \multirow{2}{*}{$\begin{array}{l}\text { Lauflänge } \\
(\mathrm{mm})\end{array}$} & \multicolumn{3}{|c|}{ Energiedichte $\left(\mathrm{J} / \mathrm{mm}^{2}\right)$ bei Abstand $(\mathrm{mm})$} \\
\hline & & & 0 & 5 & 10 \\
\hline \multirow[t]{2}{*}{$8 \mathrm{~mm}$} & \multirow[t]{2}{*}{ Pistole } & 57 & 0,57 & 0,20 & 0,09 \\
\hline & & 125 & 0,27 & 0,10 & 0,05 \\
\hline \multirow[t]{2}{*}{380} & \multirow[t]{2}{*}{ Revolver } & 105 & 0,75 & 0,27 & 0,13 \\
\hline & & 140 & 0,24 & 0,09 & 0,04 \\
\hline $9 \mathrm{~mm}$ PA & Pistole & 68 & 0,50 & 0,19 & 0,09 \\
\hline
\end{tabular}

dass selbst Herztreffer nicht zu einer sofortigen Handlungsunfähigkeit führen müssen, sondern dass Personen mit tödlichen Herztreffern und unter Eröffnung der linken oder beider Herzhöhlen sogar noch in der Lage waren, etliche Meter zu laufen, Waffen nachzuladen oder nach Schusserhalt ihrerseits Schüsse abzugeben.

\section{Warum sind Gas-/Schreck- schusswaffen beim absoluten Nahschuss gefährlich?}

Dass Schüsse mit Kartuschenmunition („Platzpatronen“) aus so genannten Gasund Schreckschusswaffen im Nahbereich und insbesondere beim absoluten Nahschuss lebensgefährliche Verletzungen hervorrufen können, ist mit zahlreichen wissenschaftlichen Publikationen belegt worden (Übersicht bei [20]) und auch in die jüngere Rechtsprechung eingeflossen. In diesem Zusammenhang wird immer wieder die Frage vorgebracht, warum ein Gasstrahl in der Lage ist, schwerwiegende und sogar tödliche Verletzungen hervorzurufen.

\section{จ Aufgrund seiner hohen Strömungsgeschwindigkeit wirkt der Gasstrahl wie ein quasisolides Projektil}

Entscheidend für das Gefährdungspotenzial derartiger Waffen ist der Geschwindigkeitsverlauf im freien Gasstrahl, aus dem sich die Energiestromdichte berechnen lässt $[19,8]$. Die Strömungsgeschwindigkeiten liegen bei kurzen Laufattrappen um bis zu $3500 \mathrm{~m} / \mathrm{s}$. Durch diese enormen Geschwindigkeiten resultieren bei absoluten Nahschüssen Energiestromdichten, die regelmäßig (innerhalb der kurzen Aufprallzeit) zu Energiedichten führen, die oberhalb der Grenzenergiedichte zum Durchschlagen von Haut liegen $(0,1 \mathrm{~J} /$ $\mathrm{mm}^{2}$; - Tab.3). Aufgrund dieser hohen Strömungsgeschwindigkeit des Gasstrahls (Jet) kommt diesem der Charakter eines Massenstroms und daher - zumindest im Nahbereich - die Bedeutung eines quasisoliden Projektils zu. Gerade bei Waffen mit eher kurzen Laufattrappen und insbesondere bei Pistolen ist der Gasstrahl aufgrund seiner Energiedichte in der Lage, bis zu mehrere Zentimeter in das darunterliegende Gewebe einzudringen und sogar Knochen zu durchschlagen. Erst bei Mündungsabständen von $10 \mathrm{~mm}$ und mehr ist bei keiner der bisherigen Gas- und Schreckschusswaffen sowie der erhältlichen Kartuschenmunition mit einem Durchsetzen der Haut zu rechnen. Die Augen hingegen können auch noch bei deutlich größeren Mündungsabständen irreversibel geschädigt werden; hierbei übernehmen dann v. a. Effekte durch das Feuer aus der Mündung und durch im Abbrand befindliche hoch beschleunigte Treibmittelbestandteile wesentliche Rollen.

\section{Sterilität von Geschossen}

Schon Lagarde $[13,14]$ beschrieb in seinen Arbeiten, dass ein Geschoss durch den Schuss nicht sterilisiert wird, obwohl es durch den Schussvorgang erwärmt wird. Die Temperaturerhöhung des Geschosses während des Schussvorgangs geschieht durch mehrere Faktoren: Beim Treibmittelabbrand wird der Geschossboden erhitzt und beim Einpressen des Projektils in die Felder des Laufes entsteht weitere Wärme. Die Temperaturen beider Vorgänge addieren sich. Hinzu kommt der Reibungswiderstand beim Durchgang des Projektils durch den Lauf. Nach dem Verlassen des Laufes kommt es mün- 
dungsnah zunächst offenbar noch zu einer zusätzlichen Erwärmung des Projektils durch die begleitenden heißen Treibmittelgase [16]. Unklar ist, welche Rolle die Luft spielt: Einerseits entsteht durch den Luftwiderstand Reibungswärme, andererseits ist die Luft deutlich kühler als das Projektil und führt zum Wärmeverlust durch Konvektion.

Nach Lampel u. Seitz [15] werden bei kleineren Kalibern, hartem Geschossmantel und langem Lauf bis zu 7\% der Treibmittelenergie in die Erwärmung des Projektils umgesetzt; bei größeren Kalibern mit weichem Mantelmaterial und kürzeren Läufen ist diese Erwärmung deutlich geringer ausgebildet. Für ein Vollmantelprojektil, aus einer Langwaffe verschossen, errechneten sie eine Erwärmung des Geschosses auf $170^{\circ} \mathrm{C}$. Aufgrund der hohen Geschwindigkeit der Geschosse (bei Langwaffen in der Größenordnung von 600-900 m/s) steckt das Projektil schon nach Bruchteilen von Sekunden im Endmedium, sodass die Einwirkzeit der Temperatur nicht ausreichen kann, um die Geschossoberfläche zu sterilisieren. Kontaminationsversuche, bei denen mit spezifischen Bakterien verunreinigte Projektile verschossen und anschließend im Endmedium nachgewiesen wurden, bestätigten dies $[3,26,28]$.

Die vorgenannten Überlegungen sind praktisch von untergeordneter Bedeutung, da beim Eindringen des Projektils die Bakterien der Hautoberfläche und bei bekleideten Regionen insbesondere auch der Textilien in den gesamten Wundkanal verschleppt werden $[27,11]$.

\section{Wirkung und Wirksamkeit: Beurteilung des Verletzungs- potenzials von Munition}

Bei der Beurteilung und Beschreibung des Verletzungspotenzials von Munition bzw. Waffensystemen werden die Begriffe Wirkung und Wirksamkeit sehr häufig nicht eindeutig verwendet, obwohl sie Unterschiedliches beschreiben: Die Wirkung eines Geschosses oder eines Waffensystems stellt eine individuelle Situation dar, bei der ein aus einer Waffe verschossenes Projektil eine konkrete Reaktion (Wirkung) hervorgerufen hat. Die Wirkung dieses Geschosses stellt ein Einzelereig- nis dar, das von den ballistischen Eigenschaften des Geschosses, der Treffpunktlage, dem Schusskanalverlauf und den damit verletzten Strukturen sowie dem psychischen und physischen Zustand des Getroffenen zum Zeitpunkt des Treffers abhängig ist. Die Wirksamkeit hingegen beschreibt das grundsätzliche Verletzungspotenzial, das einem Geschoss bzw. System innewohnt und ist von der Auftreffenergie des Geschosses und der Fähigkeit, diese Energie entlang des Schusskanals abzugeben $(\mathrm{J} / \mathrm{cm})$, abhängig. Allein die Wirksamkeit ist geeignet, als Grundlage zur Beurteilung der Gefährlichkeit von Munition und Waffensystemen herangezogen und unter humanitären Gesichtspunkten für die Erarbeitung von Konventionen verwendet $\mathrm{zu}$ werden, da sie eine allgemeine und objektiv messbare Größe darstellt [11].

\section{Fazit für die Praxis}

Für wissenschaftliche wundballistische Untersuchungen sind ausschließlich anerkannte Gewebesimulanzien wie Gelatine und Glyzerinseifen zu verwenden, um die Ergebnisse nachvollziehbar und vergleichbar zu machen. Da die Ballistik inund außerhalb des getroffenen Körpers den Gesetzen der Physik folgt, sollten objektive naturwissenschaftliche Methoden zur Überprüfung einer Fragestellung oder Rekonstruktion eines Ereignisses herangezogen und scheinbar evidente Annahmen sowie Vermutungen vermieden werden.

\section{Korrespondenzadresse}

Prof. Dr. M.A. Rothschild

Institut für Rechtsmedizin, Universitätsklinikum Köln (AöR)

Melatengürtel 60-62, 50823 Köln

rechtsmedizin@uk-koeln.de

Interessenkonflikt. Die Autoren geben an, dass kein Interessenkonflikt besteht.

\section{Literatur}

1. Bux R, Andresen D, Rothschild MA (2002) Elektrowaffe ADVANCEDTASER M 26. Funktionsweise, Wirksamkeit und Kasuistik. Rechtsmedizin 12: 207-213

2. Fackler ML, Malinowski JA (1988) Ordnance gelatin for ballistic studies. Am J Forensic Med Pathol 9:218-219

3. Journée C, Guy R, Piedelievre R (1930) Les projectiles, vecteurs de microbes. Ann Med Leg 10:667-672
4. Karger B (1995) Penetrating gunshots to the head and lack of immediate incapacitation. I. Wound ballistics and mechanisms of incapacitation. Int J Legal Med 108:53-61

5. Karger B, Kneubuehl BP (1996) On the physics of momentum in ballistics: can the human body be displaced or knocked down by a small arms projectile? Int J Legal Med 109:147-149

6. Karger B, Brinkmann B (1997) Multiple gunshot suicides: potential for physical activity and medico-legal aspects. Int J Legal Med 110:188192

7. Karger B (2010) Schussverletzungen durch Flinten. Rechtsmedizin 20, DOI s00194-010-0657-5

8. KneubuehIBP(1998) Physikalisch-ballistischeGrundlagen zur Wirksamkeit der Gas- und Schreckschusswaffen. Bericht Nr. 1436, Gruppe Rüstung, FA 26

9. Kneubuehl BP, Thali MJ (2003) The evaluation of a synthetic long bone structure as a substitute for human tissue in gun shot experiments. Forensic Sci Int 138:44-49

10. Kneubuehl BP (2006) Gefährlichkeit weiter Schüsse. Dtsch Waffen J 42:94-95

11. Kneubuehl BP, Coupland RM, Rothschild MA, Thali MJ (2008) Wundballistik - Grundlagen und Anwendungen, 3. Aufl. Springer, Berlin Heidelberg New York Tokio, S 175, 222, 361-364

12. Kroll MW, Ho JD (2009) TASER conducted electrical weapons: physiology, pathology, and law. Springer, Berlin Heidelberg New York Tokio

13. Lagarde LA (1892) Can a septic bullet infect a gunshot wound? NY Med J 56:458

14. Lagarde LA (1895) NY Med Rec 17:25

15. Lampel W, Seitz G (1983) Jagdballistik. NeumannNeudamm, Melsungen

16. MartyW,SigristT,WylerD(1994) Measurement of skin temperatureat the entry wound by means of infrared thermography. Am J Forensic Med Pathol 15:1-4

17. Pollak S (1982) Zur Makro- und Mikromorphologie der durch Faustfeuerwaffen erzeugten Einschusswunden. Beitr Gerichtl Med 40:493-520

18. Pollak S, Ropohl D (1991) Morphologische und morphometrische Aspekte des Kontusionsringes („Schürfsaumes") an Einschusswunden. Beitr Gerichtl Med 49:183-191

19. Rothschild MA, Kneubuehl BP (1996) Physikalische Grundlagen zur Messung von Gasdruck und Energiestrom bei Schreckschußwaffen. Arch Kriminol 198:151-159

20. Rothschild MA (1999) Freiverkäufliche Schreckschusswaffen. Lübeck, Schmidt-Römhild

21. Rothschild MA, Schneider V (2000) Gunshot wound to the head with full recovery. Int J Legal Med 113:349-351

22. Sellier K (1969) Einschussstudien an der Haut. Beitr Gerichtl Med 25:265-270

23. Sellier K (1975) Schädigungen und Tod infolge Schussverletzungen. In: Mueller B (Hrsg) Gerichtliche Medizin, 2. Aufl, Bd 1. Springer, Berlin Heidelberg New York, S 563-608

24. Sellier K (1982) Schusswaffen und Schusswirkungen I. Schmidt-Römhild, Lübeck

25. Thali M, Kneubuehl BP, Zollinger U, Dirnhofer R (2002) A study of the morphology of gunshot entrance wounds, in connection with their dynamic creation, utilizing the "skin-skull-brain model". Forensic Sci Int 125:190-194

26. ThoresbyFP,DarlowHM(1967)Themechanism of primaryinfection ofbullet wounds. BrJSurg 54:359-361

27. Tian HM, Huang MJ, Liu YQ, Wang ZG (1982) Primary bacterial contamination of wound track. Acta Chir Scand Suppl 508:265-269

28. Wolf AW, Benson DR, Shoji H et al (1978) Autosterilisation in low velocity bullets. J Trauma 18:63 